\title{
Monotonie und Randspektrum bei vollstetigen Operatoren
}

\author{
LUDWIG ELSNER
}

\author{
Vorgelegt von L. Collatz
}

\section{Zusammenfassung}

Es sei $\boldsymbol{A}$ ein vollstetiger linearer Operator eines Banachraums in sich. Es wird gezeigt, daß sich die Eigenschaft ,,es gibt eine Halbordnung mit totalem Ordnungskegel, bezüglich der $A$ monoton ist "durch Aussagen allein über diejenigen Eigenwerte von $A$, deren Betrag gleich dem Spektralradius ist, charakterisieren läßt. Ähnliche Äquivalenzaussagen gelten für irreduzible und streng-monotone Operatoren.

\section{Einleitung}

Bekanntlich läßt die Monotonie vollstetiger Operatoren $A$ in einem Banachraum $X$ gewisse Rückschlüsse auf die Eigenwerte maximalen Betrages zu. So gilt unter wenig einschränkenden Zusatzvoraussetzungen die Aussage

$\mathscr{P} 1$ : Der Spektralradius $\rho=\rho(A)$ ist Eigenwert. $\rho$ hat unter den Eigenwerten vom Betrage $\rho$ die größte Ordnung des Pols der Resolvente.

(Perron-Frobenius, Krein-Rutman [6], Schaefer [7].)

Ebenso weiß man, daß alle Eigenwerte einer stochastischen Matrix, die den Betrag 1 haben, nur lineare Elementarteiler besitzen. Weitere Aussagen dieser Art sind für irreduzible nichtnegative bzw. positive Matrizen (VARGa [9], S. 30/31) und streng-monotone Operatoren (KREIN-RutMaN [6], S. 267) möglich (s. auch BoHL [13]). In jüngster Zeit wurde auch mehrfach das inverse Problem behandelt, nämlich aus Kenntnissen über das Spektrum, insbesondere das Randspektrum, auf Monotonie des Operators zu schließen. So zeigt VANDERGRAFT [8], daß es für reelle Matrizen $A$ mit der Eigenschaft $\mathscr{P} 1$ stets eine Halbordnung mit solidem Ordnungskegel gibt, in der $A$ monoton ist. BAUER-DEUTSCHSTOER [1] und VANDERGRAFT [8] bewiesen, daß es für quasi-primitive Matrizen $A$ stets einen Kegel $K$ gibt, der von $A$ sogar in sein Inneres abgebildet wird. $A$ ist dann also streng-monoton.

In der vorliegenden Arbeit werden diese Zusammenhänge systematisch untersucht. Dabei werden durchweg lineare Operatoren eines Banachraums $X$ in sich betrachtet. Neben totalen und soliden Kegeln werden auch solche mit Ordnungseinheiten untersucht.

Zunächst zeigen wir, daß sich das Ergebnis von VANDERGRAFT übertragen läßt: Ein vollstetiger linearer Operator $A$ mit $\rho(A)>0$ hat genau dann die Eigenschaft $\mathscr{P} 1$, wenn es einen totalen Kegel $K$ gibt mit $A(K) \subset K$, wenn also $A$ bezüglich einer gewissen Halbordnung monoton ist. Ebenso läßt sich das Resultat von BAUER-DEUTSCH-STOER verallgemeinern. Es sei $\mathscr{P} 4$ die folgende Eigenschaft: $\mathscr{P} 4: \rho(A)>0$ ist einfacher Eigenwert. Für alle $\lambda \neq \rho, \lambda \in \sigma(A)$ gilt $|\lambda|<\rho$. 
Für endlichdimensionales $X$ fällt $\mathscr{P} 4$ mit der Eigenschaft "quasi-primitiv“ [1] zusammen. Es gilt allgemein: $A$ ist streng-monoton genau dann, wenn $\mathscr{P} 4$ vorliegt. Zwei weitere Eigenschaften, die jeweils stärker als $\mathscr{P} 1$, aber schwächer als $\mathscr{P} 4$ sind, werden ebenfalls betrachtet.

$\mathscr{P}$ 2: $\rho(A)$ ist Eigenwert. Alle Eigenwerte vom Betrage $\rho$ sind Pole der Resolvente der Ordnung 1.

PP 3: $\rho(A)$ ist einfacher Eigenwert. Alle Eigenwerte vom Betrage $\rho$ sind Pole der Resolvente der Ordnung 1.

Es stellt sich heraus, daß sich auch diese Eigenschaften durch geeignete Monotonie-Eigenschaften beschreiben lassen. So gilt:

$A$ hat genau dann die Eigenschaft $\mathscr{P} 2$, wenn es einen Kegel $K$ gibt, so daß $A(K) \subset K$ ist und ein Eigenvektor zu $\rho(A)$ sogar Ordnungseinheit (bzw. im Kegelinneren) ist. $A$ hat genau dann Eigenschaft $\mathscr{P} 3$, wenn es einen Kegel $K$ gibt, bezüglich dessen $A$ irreduzibel ist.

Es sind jedesmal noch weitere Äquivalenzaussagen möglich.

In 2. werden die Definitionen und benötigten Hilfsmittel zusammengestellt. Die oben erwähnten Aussagen befinden sich im dritten Abschnitt.

Eine Fragestellung, die bei der Einschließung von Lösungen von Systemen linearer Differentialgleichungen mit konstanten Koeffizienten $\dot{x}=A x$ auftaucht, wird in 4. behandelt. Die Matrizen $A$, für die $e^{t A}$ für alle $t \geqq 0$ monoton sind (mit von $t$ unabhängigem Ordnungskegel), werden hier durch Spektraleigenschaften charakterisiert. Es werden vier Ergebnisse zitiert, die den ersten vier Sätzen in Abschnitt 3 entsprechen.

Im letzten Abschnitt wird gezeigt, daß die Hauptergebnisse auf eine erheblich größere Operatorenklasse übertragen werden können, auf die „quasi-vollstetigen“ Operatoren. Dabei heißt $A$ quasi-vollstetig, wenn $A=B+V$ mit vollstetigem $V$ und $\rho(B)<\rho(A)$ ist. Genau für diese Operatoren verhält sich das Spektrum am Rande des Spektralkreises wie das Spektrum vollstetiger Transformationen.

\section{Definitionen, Hilfsmittel}

Es sei $(X,\|\|)$ ein reeller Banachraum, $(\tilde{X},\|\|)$ seine Komplexifikation. Eine Menge $K \subset X, K \neq\{0\}$ heißt Kegel, wenn $\mathscr{K} 1) \alpha K \subset K(\forall \alpha \geqq 0), K+K \subset K, \mathscr{K} 2)$ $K \cap-K=\{0\}$ und $\mathscr{K} 3) K=\bar{K}$ gilt. Die durch $K$ erzeugte Halbordnung werde mit , “ bezeichnet: $x \leqq y \Leftrightarrow y-x \in K$. Es sei $\widehat{K}=K-\{0\}$ und $\stackrel{K}{K}$ das Innere von $K$.

$K$ heißt erzeugend, wenn $X=K-K$, und total, wenn $X=\overline{K-K}$ ist. $K$ heißt normal, wenn es ein $\gamma>0$ gibt, so daß aus $x \in K, y-x \in K$ folgt $\|x\| \leqq \gamma\|y\|$. Ein Kegel mit inneren Punkten heißt solide. $p \in K$ heißt Ordnungseinheit, wenn es zu jedem $x \in X$ eine Zahl $k$ gibt, so da $\beta-k p \leqq x \leqq k p$ ist. Dann ist das Funktional

$$
\|x\|_{p}=\inf \{k:-k p \leqq x \leqq k p\}
$$

auf ganz $X$ definiert. $\|x\|_{p}$ ist eine Norm, die genau für $p \in \AA^{\circ}$ stetig ist. Ist $K$ normal, so ist auch $\|x\|$ stetig bezüglich $\|x\|_{p}$. Die beiden Normen sind genau dann äquivalent, wenn $K$ normal und $p \in K$ ist. 
Es sei $[X]$ die Algebra der linearen stetigen Operatoren von $X$ in $X$. Ist $v$ eine Norm auf $X$, so bezeichnen wir mit $v$ ebenfalls die zugeordnete Norm auf $[X]$, d.h.

$$
v(A)=\sup _{x \neq 0} v(A x) / v(x), \quad A \in[X] .
$$

Im Dualraum $X^{*}$ aller linearen stetigen Funktionale auf $X$ mit der Norm

$$
\|\varphi\|_{D}=\sup _{x \neq 0}(\varphi, x) /\|x\|, \quad x \in X, \varphi \in X^{*}
$$

sei für $M \subset X$ die Menge $M^{*}$ gegeben durch

$$
M^{*}=\left\{\varphi \in X^{*}:(\varphi, x) \geqq 0 \text { für alle } x \in M\right\} \text {. }
$$

Ist $K$ ein totaler Kegel, so ist $K^{*}$ ein Kegel in $\left(X,\|\|_{D}\right)$, der duale Kegel. $\varphi \in K^{*}$ heißt strikt-positiv, wenn $(\varphi, x)>0$ für alle $x \in \hat{R}$ ist.

Für $A \in[X]$ sei $\sigma(A)$ das Spektrum (wobei $A$ mit seiner Fortsetzung $\tilde{A} \in[X]$, die durch $A(x+i y)=A x+i A y$ gegeben ist, identifiziert wird), $\rho=\rho(A)=$ $\sup \{|\lambda|: \lambda \in \sigma(A)\}$ der Spektralradius.

Für $\lambda \in \sigma(A)$ ist der Index die kleinste natürliche Zahl $s$, für die gilt

$$
x \in \tilde{X} \wedge(A-\lambda I)^{s+1} x=0 \rightarrow(A-\lambda I)^{s} x=0
$$

( $I=$ Identität). Ist $\lambda$ Pol von $A$, so ist der Index gleich der Ordnung des Pols der Resolvente (und damit endlich) ([2], S. 573). Um eine kurze Schreibweise zu haben, definieren wir

$$
s(\lambda)= \begin{cases}\text { Index von } \lambda, & \text { falls } \lambda \text { Pol } \\ \infty, & \text { falls } \lambda \in \sigma(A), \lambda \text { kein Pol } \\ 0, & \text { sonst . }\end{cases}
$$

Ein Eigenwert $\lambda$ heißt einfach, wenn $s(\lambda)=1$ und $\operatorname{dim}\{x \in X:(A-\lambda I) x=0\}=1$ ist. Wir benötigen später:

Lemma 2.1. Ist $A \in[X]$ und $\varepsilon>0$, so existiert eine $z u\|\|$ äquivalente Norm $v$ auf $X$, so daß für die zugeordnete Norm v gilt: $v(A) \leqq \rho(A)+\varepsilon([5], \mathrm{S} .88)$.

Lemma 2.2. Ist $A_{i} \in[X], \rho_{i}=\rho\left(A_{i}\right), \varepsilon_{i}>0(i=1,2)$ und $A_{1} A_{2}=A_{2} A_{1}$, so existiert eine $z u\|\|$ äquivalente Norm $v$ auf $X$, so daß für die zugeordnete Norm $v$ auf $[X]$ gilt $v\left(A_{i}\right) \leqq \rho\left(A_{i}\right)+\varepsilon_{i}(i=1,2)$.

Beweis. Ohne Beschränkung der Allgemeinheit sei $\rho_{i}+\varepsilon_{i}=1$. Nach Lemma 2.1 existiert eine Norm $\mu$ mit $\mu\left(A_{1}\right) \leqq 1$. Wegen $\lim _{n \rightarrow \infty}\left(\mu\left(A_{2}^{n}\right)\right)^{1 / n}=\rho\left(A_{2}\right)<1$ existiert eine natürliche Zahl $s$ mit $\mu\left(A_{2}^{s+1}\right) \leqq 1 . \quad v(x)=\sum_{r=0}^{s} \mu\left(A_{2}^{r} x\right)$ hat die verlangten
Eigenschaften.

Lemma 2.3. Es sei $A \in[X], A$ vollstetig und $\rho(A)=\rho \leqq 1$. Dann sind folgende Aussagen äquivalent:

(a) $s(\lambda) \leqq 1$ für alle $\lambda$ mit $|\lambda|=1$.

(b) $\exists$ eine $z u\|\|$ äquivalente Norm $v$ auf $X$ mit $v(A) \leqq 1$.

(c) $\exists$ eine Norm $v$ auf $X$ mit $v(A) \leqq 1$.

(d) $\exists N>0$, so daß für alle $k=0,1, \ldots\left\|A^{k}\right\| \leqq N$ ist. 
Beweis. (a) $\rightarrow$ (b): Es existiert (s. etwa [6], S. 280) die Darstellung

$$
A=A_{0}+\sum_{j=1}^{n} \varepsilon_{j} z_{j} \varphi_{j}
$$

mit $\rho\left(A_{0}\right)<1,\left|\varepsilon_{j}\right|=1, A z_{i}=\varepsilon_{i} z_{i}, A^{*} \varphi_{i}=\varepsilon_{i} \varphi_{i}$ und $\left(\varphi_{i}, z_{j}\right)=\delta_{i j}(i, j=1, \ldots, n)$. Dabei ist auch $n=0$, d.h. $A=A_{0}$ nicht ausgeschlossen. Sei $P_{0}=I-\sum_{j=1}^{n} z_{j} \varphi_{j}$. Es existiert nach Lemma 2.1 eine Norm $\mu$ mit $\mu\left(A_{0}\right) \leqq 1 . v(x)=\mu\left(P_{0} x\right)+\sum_{j=1}^{n}\left\|z_{j}\left(\varphi_{j}, x\right)\right\|$
hat dann die geforderten Eigenschaften.

$(b) \rightarrow(c)$ und $(b) \rightarrow(d)$ sind trivial.

$(c) \rightarrow(a)$ und $(d) \rightarrow(a)$ : Ist $s(\lambda) \geqq 2$ für ein $|\lambda|=1$, so existieren $u, v \in X, u, v \neq 0$, mit $A v=\lambda v, A u=\lambda u+v$ und daher $A^{r} u=r \lambda^{r-1} v+\lambda^{r} u$ für $r=1,2, \ldots$. Es folgt $\left\|A^{r} u\right\| \geqq r\|v\|-\|u\|$ bzw. $v(u) \geqq v\left(A^{r} u\right) \geqq r v(v)-v(u)$, ein Widerspruch gegen $(d)$ bzw. (c).

Dieser Satz ist für $X=R^{n}$ bekannt, s. etwa [4]. Ist $K$ ein Kegel in $X, A \in[X]$ und $A(K) \subset K$, so heißt $A$ monoton (bezüglich der von $K$ erzeugten Halbordnung). Eine fundamentale Aussage über monotone Operatoren ist

Satz 2.1 (Krein-Rutman-Schaefer *). Es sei $K$ total, A $[X]$ vollstetig, monoton und $\rho=\rho(A)>0$. Dann gilt

(a) $\rho$ ist Eigenwert.

(b) $s(\lambda) \leqq s(\rho)$ für $|\lambda|=\rho$.

(c) $\exists z \in \hat{K}, \varphi \in \hat{K}^{*}$ mit $A z=\rho z, A^{*} \varphi=\rho \varphi$.

Ein monotoner Operator $A$ heißt streng-monoton, wenn es zu jedem $x \in \hat{K}$ ein $n=n(x)$ gibt, so daß $A^{n} x$ Ordnungseinheit ist. Ein monotoner Operator $A$ heiBt irreduzibel, wenn für ein $\lambda>\rho(A)$ und alle $x \in R$ gilt: $A(\lambda I-A)^{-1} x$ ist Ordnungseinheit.

\section{Monotonie und Randspektrum}

Satz 2.1 besagt, daß vollstetige lineare Operatoren $A$ mit $\rho(A)>0$, die einen totalen Kegel invariant lassen, $\mathscr{P} 1$ erfüllen. Wir zeigen, daß auch die Umkehrung gilt.

Satz 3.1. Ist $A \in[X], A$ vollstetig und $\rho=\rho(A)>0$, so sind folgende Aussagen gleichbedeutend:

(1) $\exists$ Kegel $K, \stackrel{\circ}{K} \neq \emptyset, A(K) \subset K$.

(2) $\exists$ Kegel $K, K$ total, $A(K) \subset K$.

(3) $\mathscr{P} 1$ ist erfüllt.

Beweis. $(I) \rightarrow(2)$ ist trivial. (2) $\rightarrow(3)$ : s. Satz 2.1 .

(3) $\rightarrow(l)$ : Wir fassen $A$ als Operator von $X$ in sich auf, identifizieren also $A$

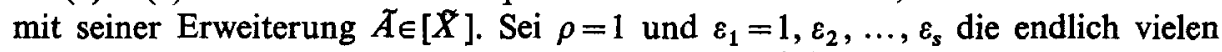
Eigenwerte von $A$, die den Betrag 1 haben. Die $\left\{\varepsilon_{i}\right\}$ sind Spektralmengen (im Sinne von DUNFORD-SCHWARTZ), es existieren also (durch Linienintegrale

* Siehe etwa Schaefer [7], S. 265. 

definierte) zugehörige Projektoren $P_{1}, \ldots, P_{s}$ und $P_{0}=I-\sum_{i=1}^{s} P_{i}([2]$, S. 568
und 573). Mit $A_{i}=A P_{i}=P_{i} A$ und $X_{i}=P_{i} \not$ gilt

$$
A=A_{0}+A_{1}+\cdots+A_{s}, \quad \tilde{X}=X_{0}+\cdots+X_{s}, \quad \rho\left(A_{0}\right)<1 .
$$

$A_{i}$ läßt $X_{i}$ invariant. Sei $i \geqq 1$. Da $\operatorname{dim} X_{i}=r_{i}$ endlich ist, besitzt $A_{i}$ eine Matrixdarstellung, die bei geeigneter Basiswahl Jordansche Normalform hat, d.h.

$$
\begin{gathered}
A_{i}=\operatorname{diag}\left(J_{1, i}, \ldots, J_{k_{l}, i}\right) \\
J_{\alpha, i}=\left(\varepsilon_{i}\right) \text { oder } J_{\alpha, i}=\left(\begin{array}{llllll}
0 & 0 & & & & 0 \\
1 & 0 & & \ldots & & 0 \\
0 & 1 & 0 & \ldots & & 0 \\
0 & 0 & & \ldots & 1 & 0
\end{array}\right)+\varepsilon_{i} I .
\end{gathered}
$$

Es gilt $\max \operatorname{dim} J_{\alpha, i}=s\left(\varepsilon_{i}\right) \leqq s(\rho)=p$.

$X_{i}$ ist also als direkte Summe von Unterräumen $N_{t, i}\left(t=1, \ldots, k_{i}\right)$ darstellbar, die bezüglich $A$ invariant sind und in denen bei der Basis $\left\{\eta_{j}\right\}$ die Abbildung $A$ so beschrieben werden kann:

$$
A \eta_{1}=\alpha \eta_{1}, A \eta_{2}=\alpha \eta_{2}+\eta_{1}, \ldots, A \eta_{k}=\alpha \eta_{k}+\eta_{k-1}
$$

$\left(\alpha=\varepsilon_{i}, k=\operatorname{dim}\left(N_{t, i}\right) \leqq p\right)$. Wir nummerieren die $N_{t, i}\left(i=1, \ldots, s ; t=1, \ldots, k_{i}\right)$ durch, nennen sie nun $N_{1}, \ldots, N_{u}\left(u=\sum_{i=1}^{s} k_{i}\right)$ und nehmen ohne Beschränkung der Allgemeinheit an, daß $N_{1}$ zum Eigenwert 1 gehört und $\operatorname{dim}\left(N_{1}\right)=p$ ist. Die Basis $\xi_{1}, \ldots, \xi_{p}$ in $N_{1}$ kann reell gewählt werden. Es gilt dann

$$
A \xi_{1}=\xi_{1}, A \xi_{2}=\xi_{2}+\xi_{1}, \ldots, A \xi_{p}=\xi_{p}+\xi_{p-1} .
$$

Sei $r \geqq 2$ fest, $q=q_{r}=\operatorname{dim}\left(N_{r}\right)(\leqq p)$ und

$$
\begin{array}{r}
K_{r}=\left\{(x, y): x=\sum_{j=1}^{p}\left(\beta_{j}+i \gamma_{j}\right) \xi_{j} \in N_{1}, y=\sum_{j=1}^{q} y_{j} \eta_{j} \in N_{r},\right. \\
\left.\left|\gamma_{j}\right| \leqq \beta_{j}, j=1, \ldots, p,\left|y_{j}\right| \leqq \beta_{j}, j=1, \ldots, q\right\} .
\end{array}
$$

Aus (3.1) und (3.2) folgt leicht, daß $A\left(K_{r}\right) \subset K_{r}$ ist. Wegen $\rho\left(A_{0}\right)<1$ existiert eine Norm $v$ mit $v\left(A_{0}\right) \leqq 1$. Wir setzen nun (mit $X_{0}=N_{0}$ )

$$
\begin{array}{r}
\tilde{K}=\left\{x \in \tilde{X}: x=x_{0}+\cdots+x_{u}, x_{i} \in N_{i}, x_{1}=\sum_{j=1}^{p}\left(\beta_{j}+i \gamma_{j}\right) \xi_{j},\right. \\
\left.\left(x_{1}, x_{r}\right) \in K_{r}, r=2, \ldots, u, v\left(x_{0}\right) \leqq \beta_{1}\right\} .
\end{array}
$$

Es folgt leicht, daß $A(\widetilde{K}) \subset \mathbb{R}$, daß $\widetilde{K}$ ein Kegel und $\mathscr{K}^{\circ} \neq \emptyset$ ist. Es ist nämlich $\tilde{x}=\sum \xi_{j} \in \stackrel{\circ}{R} . K=\widetilde{R} \cap X$ erfüllt die in (1) geforderten Bedingungen. Q.e.d. 
Nun wenden wir uns der Eigenschaft $\mathscr{P} 2 \mathrm{zu}$ :

$\mathscr{P} 2: \rho(A)$ ist Eigenwert, $s(\lambda) \leqq 1$ für $|\lambda|=\rho(A)$,

die wir folgendermaßen charakterisieren können.

Satz 3.2. Ist $X$ ein Banachraum, $A \in[X], A$ vollstetig und $\rho=\rho(A)$, so sind äquivalent:

(l) $\exists$ Kegel $K, \stackrel{\circ}{K} \neq \emptyset$ und $A(K) \subset K . \exists z \in \stackrel{\circ}{K}, \varphi \in K^{*}$ strikt-positiv und eine Zahl $\mu$ mit $A z=\mu z, A^{*} \varphi=\mu \varphi$.

(2) $\exists$ Kegel $K$ mit $A(K) \subset K$, eine Ordnungseinheit $z$ und eine Zahl $\mu$ mit $A z=\mu z$.

(3) $\rho$ ist Eigenwert. $\exists$ Norm $v$ auf $X$ mit $v(A)=\rho$.

(4) $\mathscr{P} 2$ ist erfüllt.

(5) $\rho$ ist Eigenwert. $\exists$ eine $z u\|\|$ äquivalente Norm $v$ auf $X$ mit $v(A)=\rho$.

Beweis. $(1) \rightarrow(2)$ ist trivial, (2) $\rightarrow(3)$ : Wähle $v(x)=\|x\|_{z}$ (2.1). Wegen $\rho(A) \leqq\|A\|_{z}=\|A z\|_{z}=\mu$ und $|\mu| \leqq \rho(A)$ folgt die Behauptung sofort.

Nun sei zunächst $\rho(A)>0$, und ohne Beschränkung der Allgemeinheit $\rho=1$. Dann folgt $(3) \leftrightarrow(4) \leftrightarrow(5)$ aus Lemma 2.3.

(4) $\rightarrow(l)$ : Sei $z \neq 0, \varphi \neq 0, A z=z, A^{*} \varphi=\varphi,(\varphi, z)=1$. Setze

$$
K_{\alpha}=\{x \in X: v((I-z \varphi) x) \leqq \alpha(\varphi, x)\}, \quad \alpha>0
$$

Man prüft die in ( $l$ ) geforderten Eigenschaften leicht nach.

Ist $\rho=0$, so sind alle Aussagen mit

(6) $A=0$

äquivalent. (6) $\rightarrow(1),(3) \leftrightarrow(6)$ und $(5) \leftrightarrow(6)$ sind trivial. (4) $\leftrightarrow(6)$ ersieht man etwa aus [2], S. 573, Satz $18 . \quad$ Q.e.d.

Eine leichte Folgerung ist

Corollar. Bei einer stochastischen Matrix haben die Eigenwerte vom Betrage 1 nur lineare Elementarteiler ([3], S. 102).

Nun wollen wir die Eigenschaften

$\mathscr{P} 3: \rho(A)>0$ ist einfacher Eigenwert, $s(\lambda) \leqq 1$ für $|\lambda|=\rho(A)$

und

$\mathscr{P} 4: \rho(A)>0$ ist einfacher Eigenwert. $s(\lambda)=0$ für $\lambda \neq \rho,|\lambda|=\rho$

charakterisieren.

Satz 3.3. Es sei $A \in[X], \operatorname{dim} X \geqq 2, A$ vollstetig und $\rho=\rho(A)$. Dann sind folgende Aussagen äquivalent:

(1) $\exists$ Kegel $K$ mit $\stackrel{\circ}{K} \neq \emptyset$, so daß A irreduzibel bezüglich $K$ ist.

(2) $\exists$ Kegel $K$, so daß A irreduzibel bezüglich $K$ ist.

(3) $\exists$ Kegel $K$, so daß $A(K) \subset K$ ist. $\exists$ Ordnungseinheit $z, A z=\rho z$. $\rho$ ist einfacher Eigenwert.

(4) $\mathscr{P} 3$ ist erfüllt. 
Satz 3.4. Es sei $A \in[X], A$ vollstetig und $\rho=\rho(A)$. Dann sind äquivalent:

(1) $\exists$ Kegel $K$, so daß $\stackrel{\circ}{K} \neq \emptyset$ und $A(R) \subset \stackrel{\circ}{K}$ ist.

(2) $\exists$ Kegel $K$, so daß Ax Ordnungseinheit für alle $x \in R$ ist.

(3) $\exists$ Kegel $K$, so daß A streng-monoton bezüglich $K$ ist.

(4) $\mathscr{P} 4$ ist erfüllt.

Aus technischen Gründen beginnen wir mit dem

Beweis von Satz 3.4. $(1) \rightarrow(2)$ ist trivial. $(2) \rightarrow(3)$ : Setze $n(x)=1$.

$(3) \rightarrow(4)$ : Hierfür benötigen wir eine Verschärfung von Satz 6.3 in [6], S. 267. Der angegebene Satz beinhaltet $(3) \rightarrow(4)$ unter der stärkeren Voraussetzung $\stackrel{\circ}{K} \neq \emptyset$. Es sind jedoch am dortigen Beweise nur geringe Modifikationen nötig, um ihn auch ohne diese Bedingung durchzuführen.

$(4) \rightarrow(1)$ : Ohne Beschränkung der Allgemeinheit sei $\rho=1$. A hat die Darstellung (2.2), die in diesem Falle so aussieht:

$$
A=A_{0}+z \varphi, \quad \rho\left(A_{0}\right)<1, \quad A z=z, \quad A^{*} \varphi=\varphi, \quad(\varphi, z)=1 .
$$

Es gibt nach Lemma 2.1 eine zu \|\| äquivalente Norm $v$ mit $v\left(A_{0}\right)<1$. Setze wie in Satz 3.2

$$
K_{\alpha}=\{x \in X: v((I-z \varphi) x) \leqq \alpha(\varphi, x)\}, \quad \alpha>0 .
$$

$\stackrel{\circ}{K}_{\alpha}$ besteht aus den Elementen $x$, für die die strikte Ungleichung gilt. Man rechnet leicht nach, daß $A\left(\mathbb{R}_{\alpha}\right) \subset \stackrel{\circ}{K}_{\alpha}$ ist. Q.e.d.

Beweis von Satz 3.3. (1) $\rightarrow(2)$ ist trivial. $(2) \rightarrow(3)$ : Nach Definition ist $A(K) \subset K$. Satz $3.4,(3) \rightarrow(4)$, und Satz 2.1 auf $A(\lambda I-A)^{-1}$ angewendet zeigen die Existenz von $k>0$ und einer Ordnungseinheit $z$ mit $A(\lambda I-A)^{-1} z=k z$, oder $A z=\lambda k(k+1)^{-1} z$. Da $k$ einfacher Eigenwert von $A(\lambda I-A)^{-1}$ ist, ist auch $\rho=\lambda k(k+1)^{-1}$ einfacher Eigenwert von $A$.

$(3) \rightarrow(4)$ : Siehe Satz $3.2,(2) \rightarrow(4) . \rho=0$ hat $A=0$ zur Folge. Wegen $\operatorname{dim} X \geqq 2$ ist das ein Widerspruch zur Einfachheit von $\rho$. Also ist $\rho>0$.

(4) $\rightarrow(1)$ : Es sei $\rho=1$ und $\lambda>1$ fest. $\rho\left(A(\lambda I-A)^{-1}\right)=(\lambda-1)^{-1}$ ist ebenfalls einfacher Eigenwert. Die gemeinsamen Eigenvektoren zu $A$ und $A(\lambda I-A)^{-1}$ bzw. $A^{*}$ und $A^{*}\left(\lambda I-A^{*}\right)^{-1}$ seien mit $z$ und $\varphi$ bezeichnet und auf $(\varphi, z)=1$ normiert. Es gebe eine zu \|\| äquivalente Norm $\mu$ auf $X$ mit $\mu(A) \leqq 1$ und $\mu\left((\lambda-1) A(\lambda I-A)^{-1}-z \varphi\right)<1$. Man rechnet sofort nach, daß mit

$$
K=K_{\alpha}=\{x \in X: \mu((I-z \varphi) x) \leqq \alpha(\varphi, x)\} \quad \alpha>0
$$

$A(K) \subset K$ und $A(\lambda I-A)^{-1}(\widehat{R}) \subset \stackrel{\circ}{K}$ ist, d.h. daß $A$ irreduzibel bezüglich $K$ ist. Ein solches $\mu$ kann folgendermaßen konstruiert werden: Es gilt die Zerlegung (2.2)

sowie

$$
A=z \varphi+\sum_{i=2}^{r} \varepsilon_{i} z_{i} \varphi_{i}+A_{0}, \quad\left|\varepsilon_{i}\right|=1, \quad \varepsilon_{i} \neq 1, \quad \rho\left(A_{0}\right)<1
$$

$$
(\lambda-1) A(\lambda I-A)^{-1}=z \varphi+\sum_{i=2}^{r} \frac{\lambda-1}{\hat{\lambda}-\varepsilon_{i}} \varepsilon_{i} z_{i} \varphi_{i}+(\lambda-1) A_{0}\left(\lambda I-A_{0}\right)^{-1} .
$$


$B_{0}=(\lambda-1) A_{0}\left(\lambda I-A_{0}\right)^{-1}$ ist mit $A_{0}$ vertauschbar. Da außerdem $\rho\left(A_{0}\right)<1$ sowie $\rho\left(B_{0}\right)<1$ gilt, existiert nach Lemma 2.2 eine zu \| $\|$ äquivalente Norm $v$ auf $X$ mit $v\left(A_{0}\right)<1, v\left(B_{0}\right)<1$. Mit den Projektionen $P_{1}=z \varphi, P_{i}=z_{i} \varphi_{i}(i=2, \ldots, r), P_{0}=$ $I-\sum_{i=1}^{r} P_{i}$ setzen wir

$$
\mu(x)=v\left(P_{0} x\right)+\sum_{i=1}^{r}\left\|P_{i} x\right\| .
$$

$\mu$ hat die geforderten Eigenschaften.

$\mathrm{Zu}$ den hier benutzten Begriffen „Ordnungseinheit" und ,irreduzibel“" sollen noch zwei Anmerkungen gemacht werden.

Bemerkung 1. Bekanntlich sind in einem Banachraum ([7], S. 232, Corollary 2.2) bei einem normalen Kegel alle Ordnungseinheiten innere Punkte. Da jedoch in den Sätzen 3.1-3.4 nirgends $K$ als normal vorausgesetzt wird, ist die Äquivalenz von Aussagen über Kegel mit inneren Punkten und entsprechenden Aussagen über Kegel mit Ordnungseinheiten keine unmittelbare Folge des obigen Ergebnisses.

Bemerkung 2. Die hier verwendete Definition von ,,irreduzibel" stimmt für $\stackrel{\circ}{K} \neq \emptyset$ (oder für $K$ normal) mit der von SchafFer ([7], S. 269) gegebenen überein, ist i.allg. jedoch stärker. Wir führen eine Charakterisierung dieses Begriffes an, die im Gegensatz zu einem Ergebnis von Schaefer keine Verbandsstruktur in $X$ voraussetzt. Für $X=R^{n}$ findet sich eine analoge Aussage in [8]. Für $y \in K$ sei $L_{y}$ der vom "Intervall“" $\{x: 0 \leqq x \leqq y\}$ erzeugte abgeschlossene lineare Teilraum. Dann gilt:

Satz 3.5 (ohne Beweis). Sei $\stackrel{\circ}{K} \neq \emptyset$. Dann ist $A$ irreduzibel genau dann, wenn $A$ monoton ist und aus $A\left(L_{y}\right) \subset L_{y}$ folgt: $L_{y}=X$ oder $L_{y}=\{0\}$.

\section{Monotonie von $e^{t A}$}

In diesem Abschnitt sei $X=R^{n}$ und $A$ eine reelle $n \times n$-Matrix. Die Monotonie von $e^{t A}$ für alle $t \geqq 0$ können wir so interpretieren: Sei $x_{0} \geqq 0$ und $x(t)$ die Lösung der Anfangswertaufgabe $\dot{x}=A x, x(0)=x_{0}$. Dann gilt für alle $t \geqq 0: x(t)=$ $e^{t A} x_{0} \geqq 0$. Die Bahnkurve verläuft also ganz im positiven Kegel.

Ist $e^{t A}$ monoton, so ergeben sich aus den Sätzen des vorigen Abschnitts sofort notwendige Bedingungen für die Eigenwerte von $A$. Es läßt sich jedoch auch zeigen, daß diese hinreichend sind. Im einzelnen erhalten wir vier den Sätzen 3.1 - 3.4 entsprechende Äquivalenzaussagen.

Es sei $\xi=\xi(A)=\max \{\operatorname{Re}(\mu): \mu \in \sigma(A)\}$, ([9], S. 258).

Satz 4.1. Folgende Aussagen sind äquivalent:

(1) $\xi(A)$ ist Eigenwert und $s(\mu) \leqq s(\xi)$ für $\operatorname{Re}(\mu)=\xi$.

(2) $\exists$ solider Kegel $K$ mit $e^{t A}(K) \subset K$ für alle $t \geqq 0$.

Satz 4.2. Folgende Aussagen sind äquivalent:

(1) $\xi(A)$ ist Eigenwert und $s(\mu) \leqq 1$ für $\operatorname{Re}(\mu)=\xi$.

(2) $\exists$ solider Kegel $K$ mit $e^{t A}(K) \subset K$ für alle $t \geqq 0$. Es existiert Eigenvektor $z u$ $\rho\left(e^{t \boldsymbol{A}}\right)$ in $\stackrel{\circ}{K}$. 
Satz 4.3. Folgende Aussagen sind äquivalent:

(1) $\xi(A)$ ist einfacher Eigenwert und $s(\mu) \leqq 1$ für $\operatorname{Re}(\mu)=\xi$.

(2) $\exists$ solider Kegel $K$, so daß $e^{\text {tA }}$ für alle $t>0$ irreduzibel bezüglich $K$ ist.

Satz 4.4. Folgende Aussagen sind äquivalent:

(1) $\xi(A)$ ist einfacher Eigenwert. Alle übrigen Eigenwerte von $A$ haben einen kleineren Realteil.

(2) $\exists$ solider Kegel $K$, so daß $e^{t A}(\hat{K}) \subset \stackrel{\circ}{K}$ für alle $t>0$ ist.

Die Beweise sollen hier nur kurz angedeutet werden. Die Richtung $(2) \rightarrow(I)$ ist mit Hilfe der jeweiligen Sätze 3.1 - 3.4 einfach zu beweisen. Für die Umkehrung ergeben diese Sätze zunächst nur die Existenz von Kegeln $K(t)$ mit den zusätzlichen Eigenschaften. Es ist nun möglich zu zeigen, daß $K(t)$ unabhängig von $t$ gewählt werden kann. Dabei wird z. B. folgender Hilfssatz verwendet:

Lemma 4.1 (ohne Beweis). Ist $\lambda>\xi(A)$, so existiert eine Norm $v$ auf $X$, so daß $v\left(e^{t A}\right)<e^{\lambda t}$ für alle $t>0$ ist.

Satz 4.4, $(1) \rightarrow(2)$, gestattet auch einen schnelleren Beweis. Nach Satz 3.4 existiert $\alpha>0$ und ein Kegel $K$ mit $(A+\alpha I)(\widehat{R}) \subset \stackrel{\circ}{K}$. Daher ist auch $e^{t A}(\widehat{R})=$ $e^{-\alpha t} e^{t(A+\alpha I)}(\hat{K}) \subset \stackrel{\circ}{K}$ für $t>0$.

\section{Erweiterungen}

Die folgende Eigenschaft $\mathscr{Q}$ hat sich vorne als wichtig für unsere Betrachtungen erwiesen. Bis auf $\rho(A)>0$ ist sie für vollstetige Operatoren stets erfüllt.

2: $\rho(A)>0$. Für $\lambda \in \sigma(A),|\lambda|=\rho(A)$ gilt: $\lambda$ ist Pol von $A$; ist $P_{\lambda}$ der zugehörige

Projektor, so ist $P_{\lambda} X$ endlichdimensional; die Dimension der Nullräume von

$A-\lambda I$ und $A^{*}-\lambda I$ sind gleich und endlich; $(A-\lambda I) x=y$ hat genau dann eine

Lösung $x$, wenn aus $\left(A^{*}-\lambda I\right) \varphi=0$ folgt: $(\varphi, y)=0$.

Wir nennen $A \in[X]$ quasi-vollstetig, wenn es eine Zerlegung $A=B+V$ gibt, so daß $V$ vollstetig und $\rho(B)<\rho(A)$ ist (z. B. $A=\lambda I+V, \rho(A)>\lambda$ ).

Satz 5.1. $A \in[X]$ erfüllt $\mathscr{Q}$ genau dann, wenn $A$ quasi-vollstetig ist.

Beweis. $A$ erfülle 2 . Sind $\varepsilon_{1}, \ldots, \varepsilon_{n}$ die endlich vielen Eigenwerte mit $\left|\varepsilon_{i}\right|=\rho, P_{i}$ die zugehörigen Projektoren, so ist $V=\sum P_{i} A$ endlichdimensional, also vollstetig, und $\rho(A-V)<\rho(A)$. $A$ ist also quasi-vollstetig. Sei $A$ quasi-vollstetig. Für $|\lambda|>\rho(B)$ gilt die Zerlegung $\lambda I-A=(\lambda I-B)-V$ mit stetig invertierbarem $\lambda I-B$. Für einen solchen Operator gilt die „Fredholmsche Alternative“ (s. etwa [11], S. 426, Satz 1). Die beiden letzten Aussagen von $\mathscr{Q}$ sind also erfüllt. Ist $\lambda$ mit $|\lambda|>\rho(B)$ kein Eigenwert von $A$, so ist wegen $(\lambda I-A)^{-1}=(\lambda I-B)^{-1}(I-$ $\left.V(\lambda I-B)^{-1}\right)^{-1} 1$ kein Eigenwert des vollstetigen Operators $V(\lambda I-B)^{-1}$. $\left(I-V(\lambda I-B)^{-1}\right)^{-1}$ und damit $(\lambda I-A)^{-1}$ sind also beschränkt.

Anders gewendet: Ist $\lambda \in \sigma(A),|\lambda|>\rho(B)$, so ist $\lambda$ Eigenwert von $A$ und $1 \in \sigma\left(V(\lambda I-B)^{-1}\right)$. Satz 1.9 in [12], S. 370 zeigt, da $B \lambda$ isoliert ist. $P_{\lambda} X$ ist nach einem Resultat von GoHBERG-Krein ([10], S. 202, Satz 3.2), auf $A P_{\lambda}$ und $P_{\lambda} X$ angewendet, endlichdimensional. $\lambda$ ist also Pol. Q.e.d.

Die Aussagen von Satz 2.1 bleiben auch für quasi-vollstetige Operatoren erhalten. 
Satz 5.2. Es sei $X$ ein reeller Banachraum, $A \in[X]$ quasi-vollstetig, $\rho=\rho(A)$, $K \subset X$ ein totaler Kegel und $A(K) \subset K$. Dann gilt:

(a) $\rho$ ist Eigenwert.

(b) $s(\lambda) \leqq s(\rho)$ für $|\lambda|=\rho$.

(c) $\exists z \in \hat{K}, \varphi \in \hat{K}^{*}$ mit $A z=\rho z, A^{*} \varphi=\rho \varphi$.

Beweis. (a) und $(b)$ folgen unmittelbar aus einem Ergebnis von ScHAEFER ([7], S. 264).

Um (c) zu zeigen, können wir wörtlich den ersten Teil des Beweises von Satz 6.1 in [6], S. 262 übernehmen. Dort wird die Existenz von $z$ und $\varphi$ unter der Bedingung ( $a$ ) hergeleitet. Es gehen nur die in $Q$ zusammengefaßten Eigenschaften ein. Q.e.d.

Man prüft nun unmittelbar nach, daß Lemma 2.3 und die Sätze 3.1 - 3.4 auch unter der Voraussetzung gelten, daß $A$ quasi-vollstetig ist. Die neuen Ergebnisse stellen i.allg. allerdings nur für $\rho(A)>0$ Verallgemeinerungen dar.

Die Arbeit ist eine Zusammenfassung des Hauptteils der Habilitationsschrift des Verfassers. Herrn Professor Dr. Dr. h. c. L. Collatz und Herrn Prof. Dr. R. ANsorge bin ich zu besonderem Dank verpflichtet. Ein Teil der Arbeit entstand während eines Aufenthalts beim National Research Institute for Mathematical Sciences, Pretoria, South Africa.

\section{Literatur}

1. Bauer, F. L., E. Deutsch, \& J. Stoer, Abschätzungen für die Eigenwerte positiver linearer Operatoren. Lin. Alg. and its Appl. 2, 275-301 (1969).

2. Dunford, N., \& J. T. Schwartz, Linear Operators, Part I. New York-Toronto-London: Interscience Publ., Inc. 1958.

3. GanTmacher, F. R., Applications of the Theory of Matrices. New York-London 1959.

4. Householder, A. S., The Theory of Matrices in Numerical Analysis. New York 1964.

5. Krasnoselskis, M. A., Positive Solutions of Operator Equations, Groningen 1964.

6. Krein, M. G., \& M. A. RUTMAN, Linear operators leaving invariant a cone in a Banach space. Transl. Ser. 1, vol. 10, Am. Math. Soc. 1962.

7. SchaEfer, H. H., Topological Vector Spaces. New York: Macmillan 1966.

8. VANDERGRAFT, J. S., Spectral properties of matrices which have invariant cones. SIAM 16, 1208- 1222 (1968).

9. VARGA, R. S., Matrix Iterative Analysis. Prentice-Hall, Inc. 1962.

10. GohberG, I. C., \& M. G. KreIN, The basic propositions on defect numbers, root numbers and indices of linear operators. Transl. Ser. 1, vol. 13, Am. Math. Soc. 1960.

11. Kantorowitsch, L. W., \& G. P. AkILow, Funktionalanalysis in normierten Räumen. Berlin: Akademie-Verlag 1964.

12. Kato, T., Perturbation Theory for Linear Operators. Berlin-Heidelberg-New York: Springer 1966.

13. BoHL, E., Eigenwertaufgaben bei monotonen Operatoren und Fehlerabschätzungen für Operatorgleichungen. Arch. Rational Mech. Anal. 22, 313-332 (1966).

Institut für Angewandte Mathematik der Universität Hamburg

2 Hamburg 13

Rothenbaumchaussee 67/69

Germany 